\title{
Correction to: Local Linear Estimation of the Conditional Cumulative Distribution Function: Censored Functional Data Case
}

\author{
Saâdia Rahmani and Oussama Bouanani \\ Dr. Taher Moulay University of Saida, Saida, Algeria
}

\section{Correction to: Sankhya A}

https://doi.org/10.1007/s13171-021-00276-x

In the published paper, the expression "data case" was omitted. The complete article title should be: Local linear estimation of the conditional cumulative distribution function: Censored functional data case.

The original article has been corrected. 